\title{
Facile and rapid immobilization of copper(II) bis(oxazoline) catalysts on silica: application to Diels-Alder reactions, recycling, and unexpected effects on enantioselectivity
}

\author{
Patrick O’Leary, ${ }^{\mathrm{a}}$ Nico P. Krosveld, ${ }^{\mathrm{a}}$ Krijn P. De Jong, ${ }^{\mathrm{b}}$ Gerard van Koten ${ }^{\mathrm{a}}$ \\ and Robertus J. M. Klein Gebbink ${ }^{\mathrm{a}, *}$ \\ ${ }^{a}$ Utrecht University, Debye Institute, Department of Metal Mediated Synthesis, Padualaan 8, 3584 CH Utrecht, The Netherlands \\ ${ }^{\mathrm{b}}$ Utrecht University, Department of Inorganic Chemistry and Catalysis, Sorbonnelaan 16, 3584 CA Utrecht, The Netherlands
}

Received 6 January 2004; revised 19 February 2004; accepted 1 March 2004

\begin{abstract}
Two chiral copper(II) bis(oxazoline) complexes have been immobilized on silica via electrostatic interactions using a remarkably straightforward procedure. The immobilized catalysts were tested in a standard Diels-Alder reaction and gave surprising results. Where the immobilized $\mathrm{Cu}((S, S)$-phenyl-box $)(\mathrm{OTf})_{2}$ catalyst was used, the predominant enantiomer formed was the opposite of that produced using the same catalyst in a homogeneous reaction. This is a startling result given that the only difference is the electrostatic immobilization of the catalyst on amorphous silica. The activity of the catalyst in a hetero Diels-Alder reaction was also tested. This catalyst was also recycled, successfully maintaining a similar activity to the homogeneous analogue through a number of cycles.
\end{abstract}

(c) 2004 Elsevier Ltd. All rights reserved.

\section{Introduction}

Immobilization of catalysts represents an important area of research. The ability to recycle expensive chiral species is attractive at the research and application level and, as a result, research into immobilization of catalytic species on supports is ongoing. ${ }^{1}$

Chiral copper(II) bis(oxazoline) catalysts have been applied to the homogeneous catalysis of a wide variety of reactions. ${ }^{2}$ In many cases, products of high enantiopurity have been obtained. Bis(oxazoline) ligands, however, depending on the amino acid used, can be relatively expensive to prepare and the catalysts are often used in amounts up to $10 \mathrm{~mol} \%$ or indeed greater. ${ }^{3}$ Immobilization of these catalysts thus presents a worthwhile goal, which would expand the ability to reuse them. Recently, the immobilization of a cationic

\footnotetext{
Keywords: Copper(II) bis(oxazoline); Electrostatic immobilization; Diels-Alder; Silica; Enantioselectivity reversal.

* Corresponding author. Tel.: +31-30-2533054; fax: +31-30-2523615; e-mail: r.j.m.kleingebbink@chem.uu.nl
}

chiral rhodium-phosphine hydrogenation catalyst was reported via hydrogen bonding of the triflate counter anion with the MCM-41 silica surface. ${ }^{4}$ The electrostatically immobilized catalyst had similar activity to the catalyst in homogeneous solution with good recyclability and low levels of leaching.

Immobilization of copper(II) bis(oxazoline) catalysts has been achieved in both a covalent and noncovalent manner. ${ }^{5,6}$ Typically the methods used have involved complex procedures and in some cases significant alterations to the ligand. As triflate counterions are often used in combination with copper(II) bis(oxazoline) cations we envisaged that a process similar to that employed by De Rege with a rhodium hydrogenation catalyst, ${ }^{4 a}$ and subsequently by Thomas with both rhodium and palladium hydrogenation catalysts, ${ }^{4 b}$ could be used to immobilize these catalysts. The principle advantages of this immobilization technique are: the absence of any complexity in the immobilization process, the widespread availability of the silica inorganic support (chromatography grade silica), the lack of any need to alter the catalyst or preceding ligand in any way to facilitate the immobilization and the rapidity with which the immobilization can be completed. 


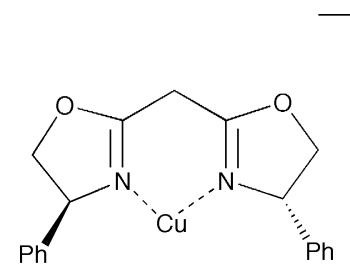

1<smiles>CCOCCO</smiles>

2

Figure 1. Copper(II) bis(oxazolines) used in this study.

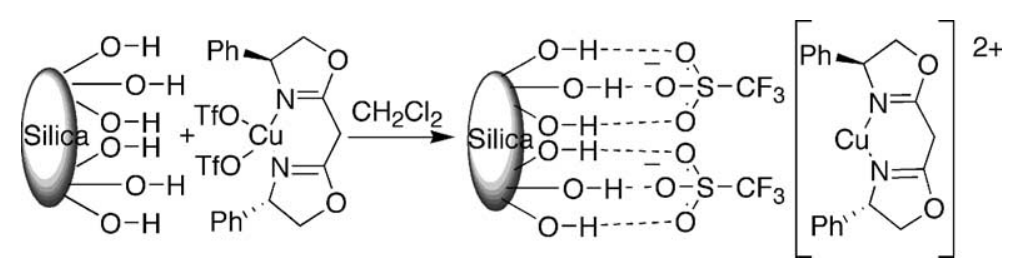

Scheme 1. Sketch of the immobilization process employed in this study (attachment via $\mathrm{H}$ bonds can be envisaged in a number of ways).

\section{Results and discussion}

The phenyl substituted copper(II) bis(oxazoline) catalyst 1 (Fig. 1) was first generated in dichloromethane from the commercially available ligand and anhydrous copper(II) triflate. Immobilization of this catalyst was achieved by addition of this solution to silica (ACROS silica gel for chromatography, pore diameter $6 \mathrm{~nm}$ ), which had been dried at $70^{\circ} \mathrm{C}$ under vacuum for $2 \mathrm{~h}$ (Scheme 1). Stirring of the homogeneous solution with the silica for a period of $2 \mathrm{~min}$ resulted in complete immobilization of the complex. The solution, which was initially a light green, was decolorized and the silica took on the same color. The solvent was decanted and the solid washed with two aliquots of dichloromethane and then stored under fresh dichloromethane until use. Typically, theoretical loadings of $1.3 \%$ copper were used in this study.

The electrostatically immobilized catalyst was initially tested in the inverse electron demand hetero Diels-Alder reaction between ethyl $(E)$-2-oxopent-3-enoate $\mathbf{3}$ and ethyl vinyl ether 4 as described by Evans using $10 \mathrm{~mol} \%$ of catalyst (Scheme 2). ${ }^{2 \mathrm{~d}}$ The major product of the reaction was the endo product 5 and the major enantiomer was the $(2 S, 4 S)$ product. ${ }^{\dagger}$ At $0{ }^{\circ} \mathrm{C}$, the reaction

\footnotetext{
${ }^{\dagger}$ Representative experimental procedure: To $10 \mathrm{~mol} \%$ of the catalyst $\mathbf{1}$ (homogeneous or heterogeneous as appropriate) in dichloromethane (5 mL) was added $30 \mathrm{mg}$ of dry $4 \AA$ molecular sieves and ethyl $(E)-2$ oxopent-3-enoate $3\left(27 \mathrm{mg}, 1.90 \times 10^{-4} \mathrm{~mol}\right)$ in dichloromethane $(2 \mathrm{~mL})$. This mixture was then allowed to equilibrate at the reaction temperature at which point ethyl vinyl ether $4(0.075 \mathrm{~mL}$, $7.84 \times 10^{-4} \mathrm{~mol}$ ) was added via syringe. The reaction mixture was stirred until completion at which point it was filtered through a short silica column (where a solid catalyst was used the reaction mixture was first decanted from the solid). The volatiles were removed under vacuum. Reaction completion was confirmed by ${ }^{1} \mathrm{H}$ NMR spectroscopy and the enantiomeric excess of the product measured by HPLC on a Chiralpak OD column. Detection was at $254 \mathrm{~nm}$. Absolute configuration was assigned by comparison with the work of Evans et al. ${ }^{2 \mathrm{~d}}$ Recovered solid catalysts were washed with two aliquots of dichloromethane before reuse.
}

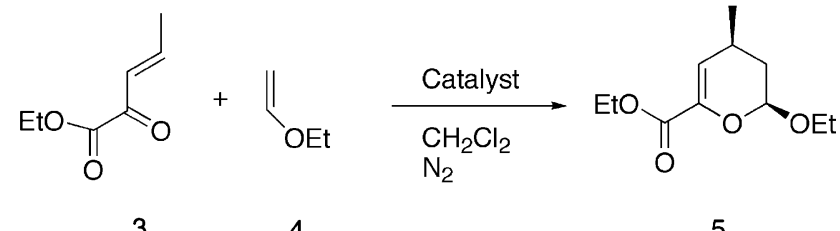

Scheme 2. Diels-Alder reaction between ethyl (E)-2-oxopent-3-enoate and ethyl vinyl ether catalyzed by electrostatically immobilized $\mathrm{Cu}$ (II) catalyst.

went to completion within $30 \mathrm{~min}$ and the product 5 was obtained in $18 \%$ ee. To compare the performance of the electrostatically immobilized catalyst with that of the catalyst in homogeneous solution, a temperature profile of the enantiopurity of the product formed in the reaction was obtained. These results are shown graphically in Figure 2. Interestingly there is little difference between the performance of the catalyst in solution and the same catalyst electrostatically immobilized on silica. The enantiomeric excess of the product formed in the reaction was slightly reduced in the case of the supported catalyst compared to the solution reaction but the most striking element of this study was the retention of activity on reuse, indeed in some cases a higher ee was observed during the second cycle. The supported catalyst was found to retain its activity for four cycles. Whereas most of the reactions conducted for this study used $10 \mathrm{~mol} \%$ of the catalyst, when $5 \mathrm{~mol} \%$ was used, a similar performance was observed both in terms of ee and recyclability.

Next, the tert-butyl copper(II) bis(oxazoline) 2 was immobilized by the same method and the resulting heterogenized catalyst was tested in the same DielsAlder reaction (see Table 1). This catalyst has been described as effective for this reaction ${ }^{2 \mathrm{~d}}$ and indeed the homogeneous reaction at room temperature gave a product with a $56 \%$ ee [the major enantiomer has the configuration $(2 R, 4 R)$ ]. The corresponding electrostatically immobilized catalyst also gave a rapid reaction, 
ee of the major endo product vs. temperature

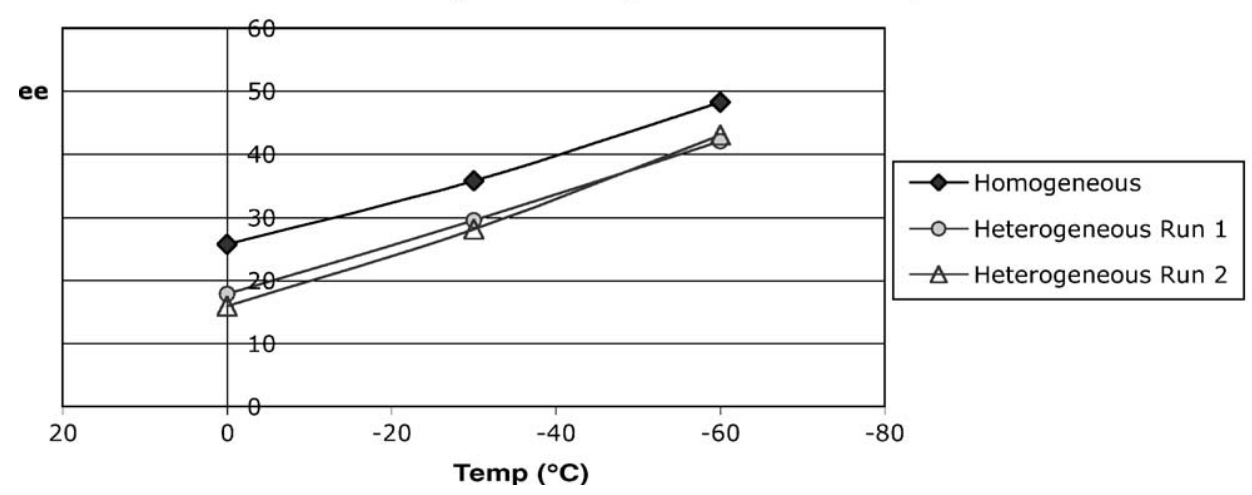

Figure 2. Results for the hetero Diels-Alder reaction between ethyl (E)-2-oxopent-3-enoate and ethyl vinyl ether catalyzed by $\mathbf{1}$ (homogeneous and heterogeneous). The ee is that of the major endo product.

Table 1. Results for the hetero Diels-Alder reaction between ethyl $(E)$ 2-oxopent-3-enoate and ethyl vinyl ether catalyzed by $\mathbf{2}$ (homogeneous and heterogeneous)

\begin{tabular}{llccll}
\hline No. & $\begin{array}{l}\text { Catalyst } \\
\text { type }\end{array}$ & Cycle & $T\left({ }^{\circ} \mathrm{C}\right)$ & $\begin{array}{l}\text { Time for } \\
\text { completion }\end{array}$ & Ee $(\%)^{\mathrm{a}}$ \\
\hline 1 & Homo & & 20 & $40 \mathrm{~min}$ & 56 \\
$2^{\mathrm{b}}$ & Homo & & -78 & & 95 \\
3 & Hetero & 1 & 20 & $20 \mathrm{~min}$ & 24 \\
4 & Hetero & 2 & 20 & $12 \mathrm{~h}$ & 17 \\
5 & Hetero & 1 & $-78->-5$ & $16 \mathrm{~h}$ & $41^{\mathrm{c}}$ \\
\hline
\end{tabular}

${ }^{\text {a }}$ Determined by HPLC on a Chiralpak OD column and detection at $254 \mathrm{~nm}$. The major enantiomer has the configuration $(2 R, 4 R)$ (assigned by analogy to the work of Evans). ${ }^{2 \mathrm{~d}}$

${ }^{\mathrm{b}}$ As reported by Evans et al. ${ }^{2 \mathrm{~d}}$

${ }^{\mathrm{c}}$ Only $25 \%$ conversion.

however, the ee of the resulting product was only $24 \%$. The catalyst was found to change color during the reaction resulting in a brown solid being recovered after the reaction. The recycled catalyst was found to be much less active and the enantiomeric excess of the product in a second cycle was poor. The rate of reaction also fell in the second cycle. A reaction conducted overnight, using freshly prepared immobilized catalyst, with an initial temperature of $-78{ }^{\circ} \mathrm{C}$ rising to $-5^{\circ} \mathrm{C}$ was found to be only $25 \%$ complete and gave product with an ee of $42 \%$ (Table 1, entry 5). It is quite evident from these results that the behavior of the immobilized tert-butyl catalyst is very different to that of its phenyl analogue. The phenyl-substituted catalyst maintained its activity, enantioselectivity and was highly recyclable, whereas the tert-butyl catalyst was obviously less stable and a less effective catalyst when immobilized by this technique.

Next, we turned our attention to the Diels-Alder reaction between $N$-acryloyloxazolidinone $\mathbf{6}$ and cyclopentadiene 7 (Scheme 3). This reaction is a well-known test reaction for enantioselective catalysts of the Diels-Alder reaction. ${ }^{7}$ The reaction was conducted at $-30{ }^{\circ} \mathrm{C}$ in dichloromethane using an excess of the cyclopentadiene. The endo diasteromer $\mathbf{8}$ is known to be the major product of this reaction and we determined the enantioselectivity of the catalyst in the synthesis of this product.

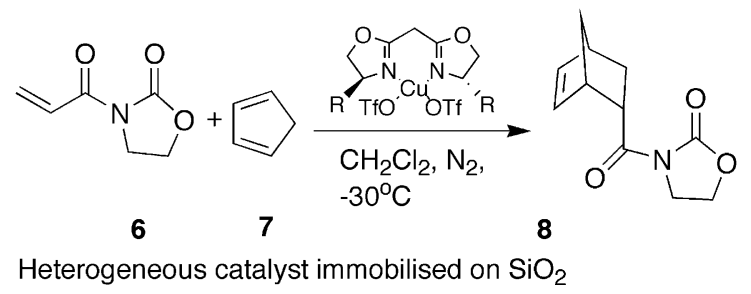

Scheme 3. Diels-Alder reaction between acryloyloxazolidinone $\mathbf{6}$ and cyclopentadiene $\mathbf{7}$ catalyzed by $\mathbf{1}$ and $\mathbf{2}$ (homogeneous and heterogeneous).

The results obtained in this study are summarized in Table 2.

We were surprised to note a very unusual phenomenon in this study. When catalyst $\mathbf{1}$ was used in homogeneous solution an ee of $20 \%$ was observed. The same catalyst, immobilized electrostatically to a silica support, gave an ee of $33 \%$. Most surprising is that the major enantiomer of the product in the homogeneous reaction is $S$ and in the heterogeneous reaction the major enantiomer is the $R$ (assigned by analogy to the work of Evans et al. ${ }^{8}$ ). This result means that using the same enantiomer of the ligand we can produce different enantiomers of the product on the basis of the interaction of the catalyst with an achiral support.

A reversal of this type has been previously observed, when a magnesium bis(oxazoline) catalyst was treated with water. ${ }^{9}$ In the anhydrous case, a tetrahedral transition state is envisaged and in the hydrated case, the transition state is thought to be octahedral. This change in co-ordination chemistry gives rise to the reversal of enantioselectivity. A related explanation could be envisaged in the general case of copper catalysts, but hydrated species have not been reported to give a reversal in enantioselectivity for copper bis(oxazolines). Thus it appears that the nature of the interaction with

\footnotetext{
\#sing the homogeneous catalyst the endo: exo ratio was $\sim 90: 10$ however the corresponding heterogeneously catalyzed reaction gave the product with $\sim 80: 20$.
} 
Table 2. Results for the Diels-Alder reaction between acryloyloxazolidinone $\mathbf{6}$ and cyclopentadiene $\mathbf{7}$ catalyzed by $\mathbf{1}$ and $\mathbf{2}$ (homogeneous and heterogeneous)

\begin{tabular}{llllll}
\hline Temperature $\left({ }^{\circ} \mathrm{C}\right)$ & Catalyst, $10 \mathrm{~mol} \%$ & $\mathrm{R}$ & Conversion $(\%)$ & Ee $(\%)$ & Major enantiomer $^{\mathrm{a}}$ \\
\hline-30 & Homogeneous & $\mathrm{Ph}$ & 100 & 20 & $S$ \\
-30 & Heterogeneous & $\mathrm{Ph}$ & 100 & 33 & $R$ \\
-50 & Homogeneous & ${ }^{t} \mathrm{Bu}$ & 50 & 59 & $S$ \\
-50 & Heterogeneous & ${ }^{t} \mathrm{Bu}$ & 43 & 57 & $S$ \\
\hline
\end{tabular}

${ }^{\mathrm{a}}$ Of the major endo diastereomer. Determined by HPLC on a Chiralpak OD column and detection at $215 \mathrm{~nm}$. The absolute configuration was assigned by analogy to the work of Evans et al. ${ }^{8}$

the silica in our case results in a different reactivity of the copper species compared to its hydrated analogue.

\section{Conclusion}

The electrostatically immobilized bis(oxazoline) catalysts prepared in this study are the first to be immobilized on silica by this method. A hetero Diels-Alder reaction has been catalyzed and gave products in up to $42 \%$ ee. This is the highest ee of which we are aware in a Diels-Alder cycloaddition obtained by reaction of a noncovalently immobilized bis(oxazoline) catalyst. It is apparent from our results that careful selection of the ligands used, is important. The phenyl substituted bis(oxazoline) 1 maintained its activity on immobilization and through a number of recycles, whereas this was not the case for catalyst 2 .

In a standard homo Diels-Alder reaction, interaction of the catalyst with an achiral inorganic support altered its reactivity sufficiently to produce the previously less favored enantiomer in excess. This obviously has very significant implications in a wide area of chemistry as traditionally one enantiomer is much more expensive to produce than the other. This development may allow use of the easily available chiral pool to produce not only straightforward products, but also the less available opposite enantiomers. Such a simple method, which offers such flexibility in the enantiomer produced is of considerable interest and we will be studying this effect in detail.

\section{Acknowledgements}

This work was supported by the Netherlands' Research School Combination-Catalysis (NRSC-C).

\section{References and notes}

1. Song, C. E.; Lee, S.-G. Chem. Rev. 2002, 102, 3495-3524.

2. (a) Fache, F.; Schulz, E.; Tommasino, M. L.; Lemaire, M. Chem. Rev. 2000, 100, 2159-2231; (b) Evans, D. A.; Faul, M. M.; Bilodeau, M. T.; Anderson, B. A.; Barnes, D. M. J. Am. Chem. Soc. 1993, 115, 5328-5329; (c) Evans, D. A.; Johnson, J. S. Acc. Chem. Res. 2000, 33, 325-335; (d) Evans, D. A.; Johnson, J. S.; Olhava, E. J. J. Am. Chem. Soc. 2000, 122, 1635-1649; (e) Evans, D. A.; Burgey, C. S.; Paras, N. A.; Vojkovsky, T.; Tregay, S. W. J. Am. Chem. Soc. 1998, 120, 5824-5825.

3. Denmark, S. E.; Nakajima, N.; Nicaise, J.-C.; Fauchner, A.-M.; Edwards, J. P. J. Org. Chem. 1995, 60, 4884 4892.

4. (a) De Rege, F. M.; Morita, D. K.; Ott, K. C.; Tumas, W.; Broene, R. D. Chem. Commun. 2000, 1797-1798; (b) Raja, R.; Thomas, J. M.; Jones, M. D.; Johnson, B. F. G.; Vaughan, D. E. W. J. Am. Chem. Soc. 2003, 125, 1498214983.

5. (a) Burguete, M. I.; Fraile, J. M.; García, J. I.; GarcíaVerdugo, E.; Herrerías, C. I.; Luis, S. V.; Mayoral, J. A. J. Org. Chem. 2001, 66, 8893-8901; (b) Rechavi, D.; Lemaire, M. J. Mol. Catal. A: Chem. 2002, 182-183, 239247; (c) Hallman, K.; Moberg, C. Tetrahedron: Asymmetry 2001, 12, 1475-1478; (d) Chow, H.-F.; Mak, C. C. J. Org. Chem. 1997, 62, 5116-5127; (e) Clarke, R. J.; Shannon, I. J. Chem. Commun. 2001, 1936-1937; (f) Corma, A.; Garcia, H.; Moussaif, A.; Sabater, M. J.; Zniber, R.; Redouane, A. Chem. Commun. 2002, 1058-1059.

6. (a) Fraile, J. M.; García, J. I.; Harmer, M.; Herrerías, C. I.; Mayoral, J. A. J. Mol. Catal. A: Chem. 2001, 165, 211-218; (b) Fernández, A. I.; Fraile, J. M.; García, J. I.; Herrerías, C. I.; Mayoral, J. A.; Salvatella, L. Catal. Commun. 2001, 2, 165-170; (c) Fraile, J. M.; García, J. I.; Mayoral, J. A.; Tarnai, T. Tetrahedron: Asymmetry 1998, 9, 3997-4008; (d) Fraile, J. M.; García, J. I.; Mayoral, J. A.; Tarnai, T. Tetrahedron: Asymmetry 1997, 8, 2089-2092.

7. (a) Owens, T. D.; Souers, A. J.; Ellman, J. A. J. Org. Chem. 2003, 68, 3-10; (b) Comprehensive Asymmetric Catalysis; Jacobsen, E. J., Pfaltz, A., Yamamoto, H., Eds.; Springer: New York, 1999.

8. Evans, D. A.; Miller, S. J.; Lectka, T.; von Matt, P. J. Am. Chem. Soc. 1999, 121, 7559-7573.

9. Zanoni, G.; Castronova, F.; Franzini, M.; Vidari, G.; Giannini, E. Chem. Soc. Rev. 2003, 32, 115-129. 